\title{
EEG Power Asymmetry and Functional Connectivity as a Marker of Treatment Effectiveness in DBS Surgery for Depression
}

\author{
Maher A Quraan*,1,2, Andrea B Protzner ${ }^{3,4}$, Zafiris J Daskalakis ${ }^{5,6,7}$, Peter Giacobbe ${ }^{5,8}$, Chris W Tang ${ }^{8}$, \\ Sidney H Kennedy ${ }^{5,6,8}$, Andres M Lozano ${ }^{1,2,9}$ and Mary P McAndrews ${ }^{1,2,6,10}$
}

'Krembil Neuroscience Center, University Health Network, Toronto, ON, Canada; ${ }^{2}$ Toronto Western Research Institute, University Health Network, Toronto, ON, Canada; ${ }^{3}$ Department of Psychology, University of Calgary, Calgary, AB, Canada; ${ }^{4}$ Hotchkiss Brain Institute, University of Calgary, Calgary, AB, Canada; ${ }^{5}$ Department of Psychiatry, University of Toronto, Toronto, ON, Canada; ${ }^{6}$ Institute of Medical Sciences, University of Toronto, Toronto, ON, Canada; ${ }^{7}$ Centre for Addiction and Mental Health, Toronto, ON, Canada; ${ }^{8}$ Department of Psychiatry, University Health Network, Toronto, ON, Canada; ${ }^{9}$ Department of Surgery, University of Toronto, Toronto, ON, Canada; ${ }^{10}$ Department of Psychology, University of Toronto, Toronto, ON, Canada

Recently, deep brain stimulation (DBS) has been evaluated as an experimental therapy for treatment-resistant depression. Although there have been encouraging results in open-label trials, about half of the patients fail to achieve meaningful benefit. Although progress has been made in understanding the neurobiology of MDD, the ability to characterize differences in brain dynamics between those who do and do not benefit from DBS is lacking. In this study, we investigated EEG resting-state data recorded from 12 patients that have undergone DBS surgery. Of those, six patients were classified as responders to DBS, defined as an improvement of $50 \%$ or more on the 17-item Hamilton Rating Scale for Depression (HAMD-17). We compared hemispheric frontal theta and parietal alpha power asymmetry and synchronization asymmetry between responders and non-responders. Hemispheric power asymmetry showed statistically significant differences between responders and non-responders with healthy controls showing an asymmetry similar to responders but opposite to non-responders. This asymmetry was characterized by an increase in frontal theta in the right hemisphere relative to the left combined with an increase in parietal alpha in the left hemisphere relative to the right in non-responders compared with responders. Hemispheric mean synchronization asymmetry showed a statistically significant difference between responders and non-responders in the theta band, with healthy controls showing an asymmetry similar to responders but opposite to non-responders. This asymmetry resulted from an increase in frontal synchronization in the right hemisphere relative to the left combined with an increase in parietal synchronization in the left hemisphere relative to the right in non-responders compared with responders. Connectivity diagrams revealed long-range differences in frontal/central-parietal connectivity between the two groups in the theta band. This pattern was observed irrespective of whether EEG data were collected with active DBS or with the DBS stimulation turned off, suggesting stable functional and possibly structural modifications that may be attributed to plasticity.

Neuropsychopharmacology (2014) 39, 1270-128I; doi: I0.1038/npp.2013.330; published online 8 January 2014

Keywords: Deep brain stimulation; major depressive disorder; electroencephalography; synchronization; functional connectivity.

\section{INTRODUCTION}

Major depressive disorder (MDD) affects millions worldwide. Recent studies estimate the lifetime prevalence of MDD in the United States to range between 10.4\% and 17\% (with strong variations across different ethnicities) and the annual prevalence to range between $3.9 \%$ and $6.7 \%$ (Kessler

*Correspondence: Dr MA Quraan, Krembil Neuroscience Centre, University Health Network, 399 Bathurst St., Room 4F-409, Toronto, Ontario M5T 2S8, Canada, Tel: +I 416603 5800, E-mail: mquraan@gmail.com

Received 17 April 2013; revised 30 October 2013; accepted 31 October 2013; accepted article preview online 28 November 2013 et al, 2005b; Nock et al, 2010; Williams et al, 2007). Comorbid disorders including anxiety, impulse control, and substance abuse are a common occurrence. A shortened lifespan results from various physical disorders accompanying the disease such as cardiovascular disease and diabetes, as well as suicide. Psychosocial impairment results in more years of lost productivity than any other disease (Kessler et al, 2005a, b, 2007).

MDD patients typically are treated with psychotherapy, cognitive behavior therapy, medications or electroconvulsive therapy (ECT). Those who fail to achieve remission are said to have treatment-resistant depression (TRD) and constitute $\sim 30 \%$ of MDD patients. Recently, deep brain stimulation (DBS) has been evaluated as an experi- 
mental therapy to treat TRD patients, where electrodes are implanted stereotactically into specific neuroanatomical structures in an attempt to modulate activity of neural networks that are known to be associated with depressive symptoms. A pulse generator is used to apply continuous stimulation, with parameters (signal amplitude, frequency, pulse width, and voltage) that can be specifically adjusted for each patient (Lozano et al, 2008).

Various neuroantomical structures have been used as targets for DBS including subgenual cingulate cortex (SCC) (Holtzheimer and Mayberg, 2010; Lozano et al, 2008; Neimat et al, 2008), ventral capsule/ventral striatum (Malone et al, 2009), nucleus accumbens (Kayser et al, 2011; Schlaepfer et al, 2008), inferior thalamic peduncle (Jimenez et al, 2005) and lateral habenula (Sartorius et al, 2010). A recent pilot study also assessed the safety and efficacy of DBS to the supero-lateral branch of the medial forebrain bundle in seven patients with highly refractory depression (Schlaepfer et al, 2013) with promising results. The choice of these targets is based on various sources of evidence pointing to their involvement in depressive symptoms including functional neuroimaging studies (Kennedy et al, 2007; Lakhan and Callaway, 2010; Mayberg et al, 1999), observations of reduced depressive symptoms following ablative procedures performed on MDD and obsessive compulsive disorder patients (Greenberg et al, 2003) and lesion studies in animal models (Yang et al, 2008). Although the small number of cases published to date preclude a conclusion as to which of these locations are best suited as DBS targets, the observation that successful treatment has been achieved using various targets is compatible with the hypothesis that MDD is a network disorder. In this regard, it has been suggested that the various DBS targets can be considered as nodes within this network (Mayberg, 2003; Seminowicz et al, 2004), and hence, the stimulation of a specific node propagates throughout the network. It is noteworthy that pharmacological treatments of depression and ECT affect widespread brain systems and thus even focal treatments likely need to impact broader networks to be efficacious in complex psychiatric disorders such as MDD.

Converging evidence from structural and functional neuroimaging, histology and lesion studies implicate the medial prefrontal cortex, the orbital frontal cortex and limbic regions as key nodes in a network-regulating mood. In particular, various functional neuroimaging studies have shown the SCC and the amygdala to be hyperactive, whereas regions in the frontal cortex to be hypoactive (Giacobbe et al, 2009; Mayberg et al, 1999). In particular, Mayberg et al (2005) showed the SCC to be hyperactive in TRD patients, whereas previous literature has not reported the SCC to be hyperactive in non-TRD patients. In addition, studies evaluating brain structure using MRI have shown abnormalities in frontal regions and the cingulate gyrus, specifically, reduced volume and gray matter thickness in these areas in patients with MDD and bipolar disorder (Coryell et al, 2005; Lyoo et al, 2004; Nugent et al, 2006). These regions are thought to be part of a limbic-cortical-striatalpallidal-thalamic network that is involved in emotion processing (Anderson et al, 2012; Nugent et al, 2006).

Frontal power asymmetry is a widely used EEG biomarker of depression. EEG studies in currently depressed patients often reveal reduced left-relative-to-right frontal alpha $(\alpha)$ power. Out of 26 studies on depressed adults compiled by Thibodeau et al (2006), 10 studies showed this effect within a $95 \%$ confidence limit. Other studies focused on frontal theta $(\theta)$, as $\theta$ oscillations are implicated in emotion processing, as well as other brain functions that are affected by depression including attention, learning, and memory (Bekkedal et al, 2011; Knyazev, 2007). Frontal $\theta$ power asymmetry in depressed patients has also been reported showing higher $\theta$ power in the right hemisphere relative to the left in comparison with controls (Kwon et al, 1996). Furthermore, $\theta$ power has been shown to be a predictor of response to antidepressent medications in resistant depressive disorder (Bares et al, 2008) and combined $\theta$ and $\alpha$ frontal asymmetry was shown to be a predictor of suicidal ideation during SSRI treatment (Iosifescu et al, 2008). A recent EEG pilot study pointed to frontal $\theta$ cordance (FTC) at baseline (and higher FTC after 4 weeks) as a response predictor of lower depression severity scores after 24 weeks of stimulation of the SCG (Broadway et al, 2012). Several EEG studies have attempted to correlate asymmetries in resting-state parietal $\alpha$ to depression and anxiety symptoms (Nitschke et al, 1999; Stewart et al, 2011) generally showing an inverted hemispheric relationship between parietal $\alpha$ oscillations and cortical activity (Knyazev, 2007; Thibodeau et al, 2006). These results are in agreement with recent task experiments implicating parietal $\alpha$ in the disengagement of brain regions that are not necessary for the task. Converging evidence from various studies have accumulated in support of this interpretation (Haegens et al, 2010; Mathewson et al, 2009; van Ede et al, 2011).

A large number of EEG and MEG studies have shown that oscillations in different frequency bands are related to specific brain functions (Cornwell et al, 2008; Tesche and Karhu, 2000; Urakami, 2008; van Dijk et al, 2008; van Gerven and Jensen, 2009). Additionally, there is increasing evidence that functional interactions between different brain regions are mediated by the synchronization of their oscillations. These interactions are believed to be associated with various cognitive functions as well as the integration of information in the healthy brain (Bullmore and Sporns, 2009; Stam and Reijneveld, 2007b). This synchronization is observed in oscillatory activity recorded by EEG and MEG and refers to a consistent relation between the phases of the oscillatory activity of two brain regions that can be assessed from the measured time series (Stam et al, 2009). Recent studies on a host of neurological disorders including Alzheimer's disease (de Haan et al, 2012; Tahaei et al, 2012), Parkinson disease (Babiloni et al, 2011; Bosboom et al, 2009; Stoffers et al, 2008), schizophrenia (Hanslmayr et al, 2012; Hinkley et al, 2011; Jalili et al, 2007) and epileptic seizures (Mormann et al, 2003; Stam et al, 2007a) have demonstrated a disruption in this synchronization in various frequency bands. In MDD, impairments in synchronization in both $\theta$ and $\alpha$ bands have been reported (Fingelkurts et al, 2007; LinkenkaerHansen et al, 2005).

Based on the literature discussed above, we investigated frontal $\theta$ and parietal $\alpha$ power in a group of 12 patients with TRD that underwent DBS surgery 3-6 years before the EEG recording as well as functional connectivity in the two bands. The EEG data were acquired at rest with the DBS electrodes turned on and off. Of these patients, six showed an improvement of $50 \%$ or more in their Hamilton-17 depression scale (HAMD-17) scores compared with 
Table I Demographic Patient Data

\begin{tabular}{lcccccc}
\hline $\begin{array}{l}\text { Subject } \\
\text { ID }\end{array}$ & $\begin{array}{c}\text { Age at } \\
\text { surgery } \\
\text { (years) }\end{array}$ & & $\begin{array}{c}\text { Gender } \\
\text { MDD } \\
\text { onset }\end{array}$ & $\begin{array}{c}\text { No. } \\
\text { of } \\
\text { MDEs }\end{array}$ & $\begin{array}{c}\text { Pre-DBS } \\
\text { MDE } \\
\text { duration } \\
\text { (months) }\end{array}$ & $\begin{array}{c}\text { Time } \\
\text { between } \\
\text { surgery and } \\
\text { EEG } \\
\text { (months) }\end{array}$ \\
\hline I & 45 & F & 21 & 6 & 72 & 75 \\
2 & 44 & F & 20 & 4 & 36 & 20 \\
3 & 53 & F & 22 & - & 39 & 36 \\
4 & 43 & M & 16 & 2 & 120 & 74 \\
5 & 42 & M & I7 & 7 & 26 & 51 \\
6 & 38 & F & 16 & 3 & 108 & 38 \\
7 & 50 & M & 26 & I & 288 & 29 \\
8 & 49 & F & - & - & - & 5 \\
9 & 55 & F & 21 & 4 & 192 & 25 \\
I0 & 42 & F & 25 & 8 & 156 & 37 \\
II & 44 & F & 34 & I & 108 & 22 \\
I2 & 53 & F & 33 & 2 & 48 & 43 \\
\hline
\end{tabular}

pre-DBS baseline, and thus met the criteria for clinical response. We compared the above-mentioned measures from responders to non-responders, as well as to a group of 15 healthy controls, and explored the correlation of these measures with the percentage improvement in HAMD-17 scores from baseline for the patient group. Furthermore, we compared the two sets of data taken with the DBS electrodes on and off. Our aim was to establish: 1) whether frontal $\theta$ and parietal $\alpha$ asymmetries differ between responders and non-responders and if so, whether responders would show an asymmetry pattern similar to controls and 2) whether long-term DBS stimulation results in neural asymmetry patterns that are similar, whether the stimulation is turned on or off during data acquisition.

\section{MATERIALS AND METHODS}

\section{Subjects}

Fourteen patients with DBS electrodes implanted in the SCC participated in this study. These patients were drawn from a larger sample $(N=20)$ previously reported in Lozano et al (2008). To qualify for DBS surgery, patients were determined to have TRD, be in a current major depressive episode (MDE) for duration of at least 1 year, and obtain a score of at least 20 on the HAMD-17 scale. Inclusion and exclusion criteria have been published elsewhere (Mayberg et al, 2005). Patients underwent DBS surgery 3-6 years before the EEG recordings. Data preprocessing revealed high levels of physiological and environmental artifacts over a wide time range in two patients who were subsequently excluded from any further analysis. Demographic patient data and clinical data for the remaining 12 patients are summarized in Tables 1 and 2.

We classified our patients into two categories: those who met clinical response criteria and those who did not, where clinical response indicates a reduction of more than $50 \%$ on the HAMD-17 rating scale after DBS surgery. Post surgery scores used in this study were determined on the same day as the EEG data recordings. Of the 12 patients considered in this analysis, 6 met the response criteria.

Resting-state data also were recorded from 15 healthy volunteers between the ages of 22 and 59 years (mean age $=33 \pm 10$ ).

\section{Surgical Procedure}

The SCG was identified by direct visualization on coronal MRI images. Bilateral burr holes were placed $1 \mathrm{~cm}$ anterior to the coronal suture and $2 \mathrm{~cm}$ from the midline. Microelectrode recordings were used to identify the upper and lower cortical banks and the intervening white matter of the SCG. Quadripolar DBS electrodes (Medtronic 3387, Medtronic) were implanted with the most distal contact (contact 0 on the right, contact 4 on the left) adjacent to the ventral bank of gray matter, the two central contacts (contacts 1 and 2 right, contacts 5 and 6 left) in white matter, and the uppermost contacts (contact 3 right, contact 7 left) adjacent to the upper bank of gray matter of the SCG gyrus (Lozano et al, 2008). Figure 1 shows the anatomical location of the subgenual cingulate gyrus. Electrode positions have been extensively discussed in previous publications (Hamani et al, 2009, 2011; Laxton et al, 2013; Lozano et al, 2012).

\section{Stimulation Settings}

The selection of electrode contacts for the initial settings was made based on the behavioral effects including calmness, improved mood, increased interest and increased motivation and were typically achieved within the range of 3-6 V. As part of dose finding, stimulation intensity was increased to look for adverse effects. In two patients, mental slowing occurred at high settings of $8-10 \mathrm{~V}$, particularly at the higher contacts ( 3 and 7). In patients who showed little or no acute behavioral changes, the stimulation was applied using contacts 1 and 5 set at 3.5 V. A pulse width of $90 \mu$ s was used at a frequency of $130 \mathrm{~Hz}$. DBS signal adjustment were made at follow-up visits if the patient failed to show improvement (less than a $10 \%$ reduction in the HAMD-17 score) or developed adverse effects. Changes consisted of choosing different contact pairs for stimulation or adjusting the voltage. Throughout the stimulation adjustment process, patients remained blinded as to which contact was being stimulated and the parameter settings (Lozano et al, 2008).

\section{Data Acquisition}

Subjects were asked to sit in a quiet room and to stay fully relaxed with their eyes closed. EEG data were recorded at a sample rate of $1000 \mathrm{~Hz}$ using a Neuroscan 64-channel system. Two data sets were recorded from each patient with the DBS electrodes on and off for a duration of 6 minutes/ data set. The two data sets were recorded within a $4.5-\mathrm{h}$ period with the electrodes on session recorded first. The minimum time period between the on and off recordings was $2.8 \mathrm{~h} \quad($ mean $=3.4 \pm 0.4 \mathrm{~h})$. We refer to these two conditions as the 'ON' and 'OFF' conditions. DBS stimulation was turned off right before the resting-state OFF recording commenced. 
Table 2 HAMD- 17 Scores of Patients before and after DBS Surgery and their Response Status

\begin{tabular}{lcccc}
\hline $\begin{array}{l}\text { Subject } \\
\text { ID }\end{array}$ & $\begin{array}{c}\text { HAMD-I7 Pre } \\
\text { surgery }\end{array}$ & $\begin{array}{c}\text { HAMD-I7 Post } \\
\text { surgery }\end{array}$ & $\begin{array}{c}\text { HAMD-I7\% } \\
\text { change }\end{array}$ & Response \\
\hline 1 & 26 & 1 & 96 & Yes \\
2 & 25 & 5 & 80 & Yes \\
3 & 26 & 6 & 77 & Yes \\
4 & 26 & 2 & 92 & Yes \\
5 & 24 & 8 & 67 & Yes \\
6 & 20 & 14 & 30 & No \\
7 & 28 & 24 & 14 & No \\
8 & 28 & 18 & 36 & No \\
9 & 31 & 9 & 71 & Yes \\
10 & 21 & 13 & 38 & No \\
11 & 28 & 17 & 39 & No \\
12 & 20 & 19 & 5 & No \\
\hline
\end{tabular}

\section{Data Analysis}

Preprocessing. In the offline data preprocessing, each channel was re-referenced to the average of all channels to avoid systematic effects that may arise from referencing to a particular channel, particularly in the context of synchronization analysis (Guevara et al, 2005; Nunez et al, 1997; Stam et al, 2007a). A DC offset was subtracted based on the entire time range, and bad channels were removed then interpolated from neighboring channels.

Spectral power from EEG signals is generally difficult to quantify at low and high frequencies due to ocular and muscle artefacts in these regions. Signals from ocular artefacts are orders of magnitude higher than neural signals and result in a sharp rise in spectral power at low frequencies, whereas muscle artefacts make large contributions at high frequencies. Despite the various methods available to reduce such artefacts, determining their contribution to systematic errors is difficult to achieve in data with small number of subjects. The data were therefore further bandpassed in the $2-20 \mathrm{~Hz}$ range to avoid such artifacts. The low-pass filter also served to remove artifacts from DBS stimulation, as the DBS electrodes were stimulated at a frequency of $130 \mathrm{~Hz}$ and had negligible effect (if any) on data over the frequency range of interest. It has been previously shown that bipolar DBS stimulation produce clinically insignificant artifacts (Frysinger et al, 2006). Remaining artefacts were removed manually by rejecting segments containing artefacts.

Spectral power. The cleaned data was fast Fourier transformed (FFT) to obtain the absolute spectral power for each channel in two frequency bands: $\theta(4-8 \mathrm{~Hz})$ and $\alpha$ $(8-12 \mathrm{~Hz})$. A frontal power asymmetry measure, $A_{\mathrm{F}}$, in the $\theta$ band was defined using channels F3 and F4 (see Appendix 1). A parietal $\alpha$ power asymmetry, $A_{\mathrm{P}}$, was defined similarly using channels P3 and P4. Note that odd number locations are sensors on the left hemisphere and even numbered are on the right hemisphere.

An accumulating number of EEG and MEG studies implicate $\alpha$ oscillations in hemispheric disengagement of

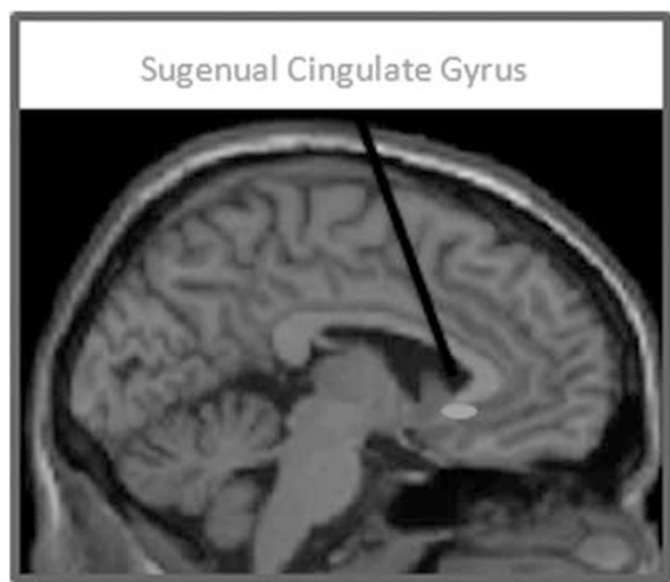

Figure I Anatomical location of the subgenual cingulate gyrus.

brain regions contralateral to those involved in task performance (Haegens et al, 2012; van Ede et al, 2011). Hence, we defined a hemispheric asymmetry computed as the weighted sum of frontal $\theta$ left-right power difference and parietal $\alpha$ right-left power difference. A mathematical formalism of these quantities is provided in the Appendix 1.

Although an asymmetry measure is limited in its ability to reveal whether the asymmetry results from an increase in right hemispheric power or a decrease in left hemispheric power, it provides advantages over measures of power, as common left-right variations in signal-to-noise ratio across subjects would be accounted for. This is particularly important when dealing with a small sample size. These variations can result from differences in scalp thickness across subjects as well as various sources of noise. In particular, frontal sources are highly influenced by ocular eye artifacts, some of which are likely to escape data cleaning.

Functional connectivity. Synchronization of chaotic systems has captured tremendous interest in the field of nonlinear dynamics and has been used in a wide range of scientific applications. Phase synchronization, a specific measure of synchronization was first introduced by Rosenblum et al (1996) and found applications to time series recorded from brain activity soon after (Mormann et al, 2000; Tass et al, 1998). Following Mormann et al (2000), we use a mean phase coherency measure of synchronization, which takes on values between 0 and 1 , indicating no synchronization and full synchronization, respectively. In comparison with amplitude-based correlation measures, phase synchronization measures are less influenced by signal-to-noise fluctuations, and as such, result in a more robust measure of functional connectivity.

In this study, we computed phase coherence between all pairs of electrodes, and then computed a mean synchronization for each electrode. Using this mean synchronization, we computed a hemispheric mean synchronization asymmetry (similar to hemispheric power asymmetry) based on the regions shown in Figure 4c. Details of these calculations are provided in the Appendix 1.

Synchronization measures also can be used to study network architecture. In the context of network theory, network diagrams are represented geometrically as a set of 
nodes (representing processing centers) and edges that represent the information flow between them. This topological representation of the geometry is known as graph theory. To this end, we created network diagrams to investigate differences in synchronization between responders and nonresponders for both the $\mathrm{ON}$ and OFF conditions.
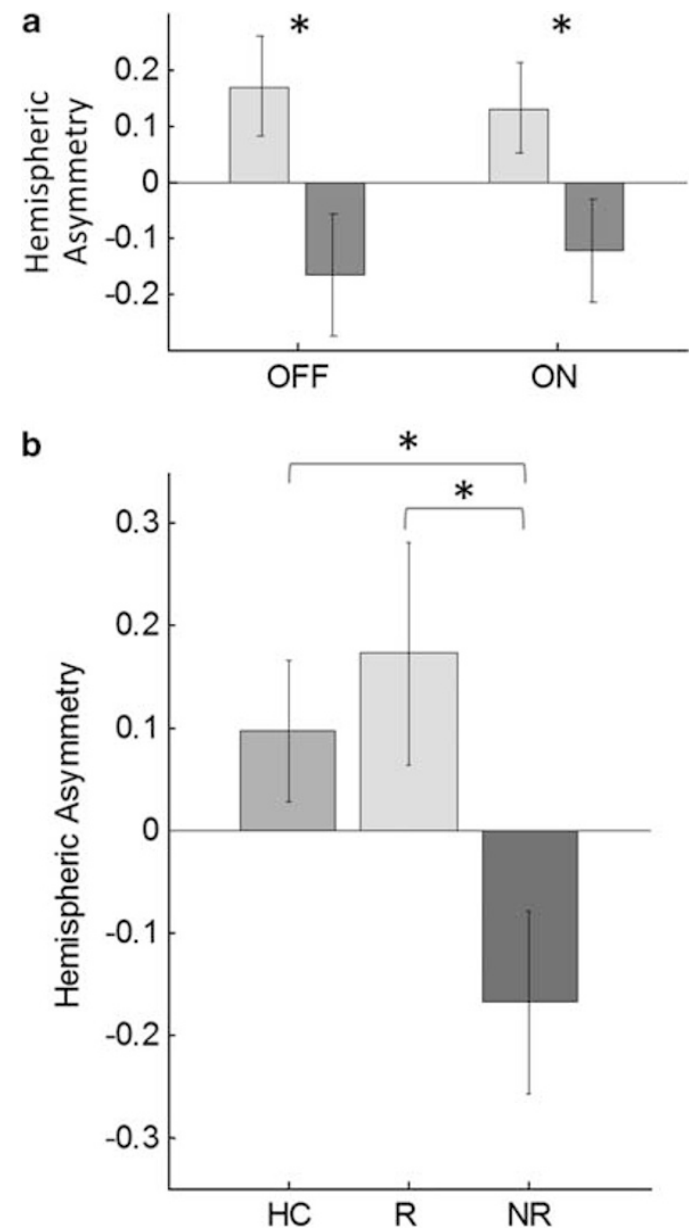

Figure 2 (a) Hemispheric asymmetry (see Equation 2 in the Appendix I) computed as the weighted sum of frontal $\theta$ hemispheric power difference and parietal $\alpha$ hemispheric power difference. (b) The same as the OFF condition in a with data from 15 healthy controls $(\mathrm{HC})$ added to the plot. Asterisks indicate $p<0.05$ on a Kruskal-Wallis test.

\section{Statistical Analysis}

Because of the small number of patients and to avoid the assumption that our samples are drawn from a normal distribution, we used the non-parametric Kruskal-Wallis test. This test is also suitable for comparing our two groups of patients to controls as the number of subjects in each group is different. The p-values for hemispheric power asymmetry for the OFF and ON conditions are reported using this test. An ANOVA was conducted for hemispheric mean synchronization asymmetry in the two frequency bands $(\theta$ and $\alpha)$. As these were statistically significant, we pursued the two bands separately and corrected for multiple comparisons.

\section{RESULTS}

\section{Power Asymmetry}

Hemispheric power asymmetry (Equation 2 in the Appendix 1) showed statistically significant differences between responders and non-responders for both the OFF $(p=0.04)$ and $\mathrm{ON}(p=0.03)$ conditions (Figure $2 \mathrm{a})$. We further compared our patient hemispheric asymmetry data with that from 15 healthy controls (Figure 2b). Healthy controls showed an asymmetry similar to responders but opposite to non-responders $(p=0.03)$. Frontal $\theta$ power asymmetry showed a positive correlation with HAMD-17 scores in the OFF $(r=0.45)$ and ON $(r=0.31)$ conditions as can be seen from Figure 3a. Parietal $\alpha$ power asymmetry, on the other hand, showed a negative correlation with HMAD17 scores in the OFF $(r=-0.55)$ and ON $(r=-0.44)$ conditions (Figure $3 \mathrm{~b}$ ). Figure 3 indicates that hemispheric asymmetry resulted from an increase in right-relative-to-left frontal $\theta$ in non-responders relative to responders and an increase in left-relative-to-right parietal $\alpha$ in non-responders relative to responders. Statistical significance was established from the comparison of responders and nonresponders. The figures displaying trends of power asymmetry with HAMD-17 scores were not used to establish statistical significance.

Although our hypothesis was focused on the $\theta$ band and its reciprocal relationship with parietal $\alpha$, we also assessed frontal $\alpha$ asymmetry, as it is widely reported
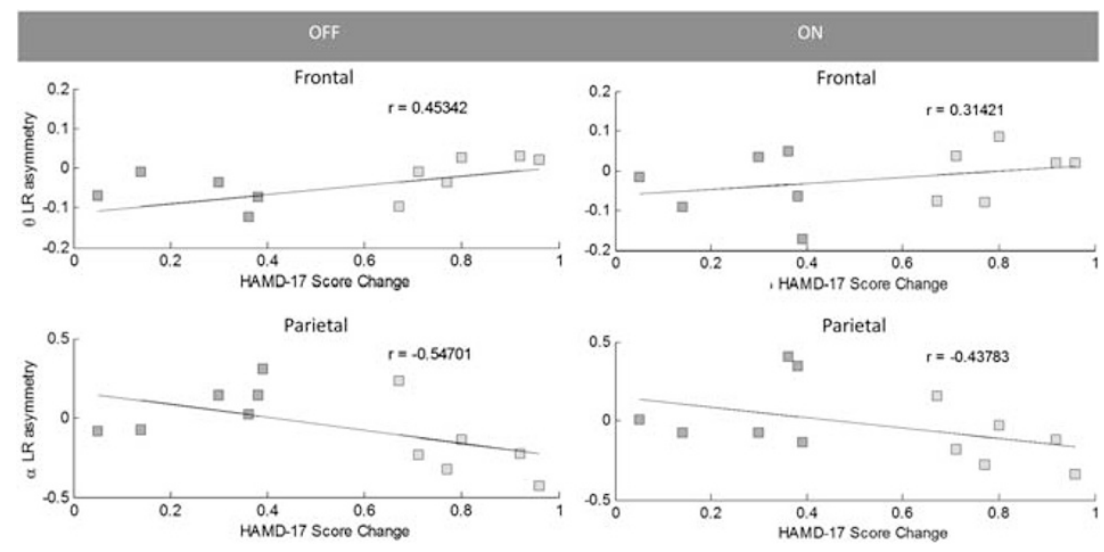

Figure 3 Top: correlation between frontal $\theta$ left-right power asymmetry and HAMD-I7 scores for the OFF (left) and ON (right) conditions. Bottom: correlation between parietal $\alpha$ left-right power asymmetry and HAMD- 17 scores. 

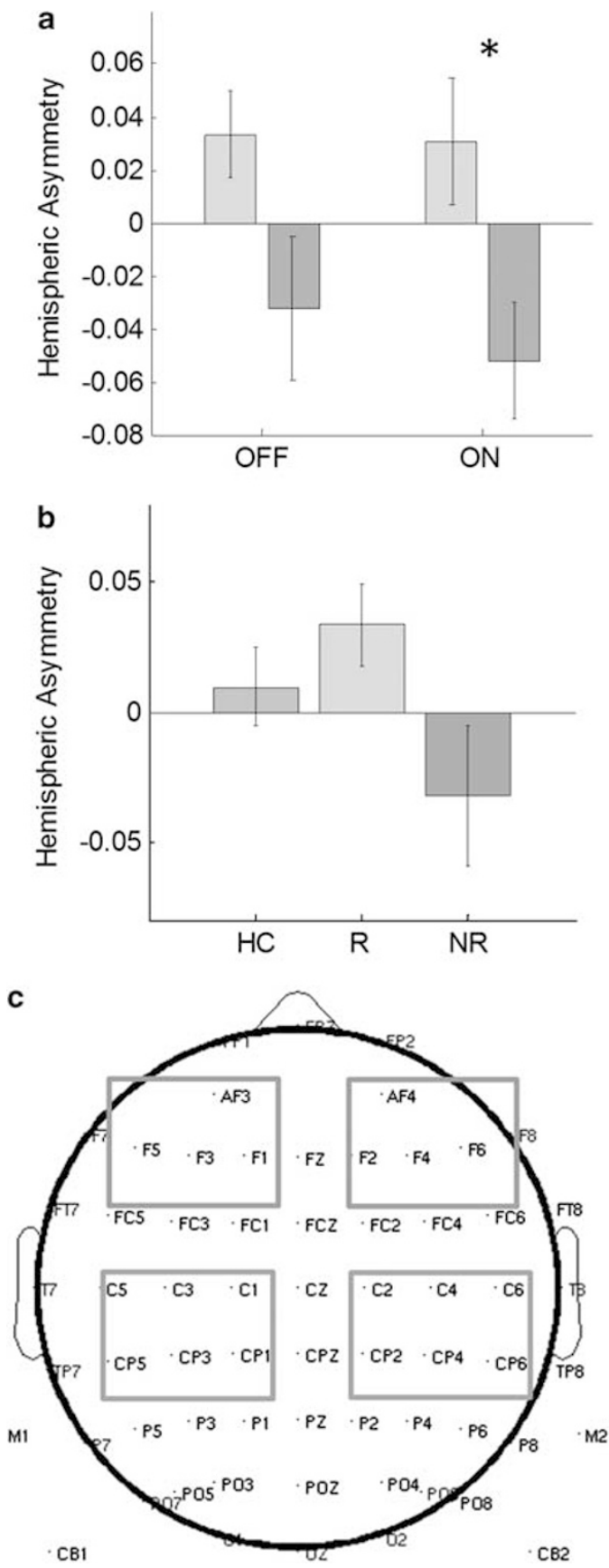

Figure 4 (a) Group-averaged hemispheric synchronization asymmetry in the $\theta$ band with the DBS electrodes OFF (left) and ON (right) for the R (response) and NR (no-response) groups as labeled on the plots. (b) Same as panel $a$, where DBS stimulation is turned off in the patient group with healthy controls $(\mathrm{HC})$ added to the plot. Asterisks indicates $p<0.05$ on a Kruskal-Wallis test. (c) EEG channel configuration used to calculate synchronization asymmetry for frontal and parietal regions. LF, left frontal; $L P$, left parietal; RF, right frontal; $R P$, right parietal.

in the literature. The results were not statistically significant.

\section{Synchronization Asymmetry}

Hemispheric mean synchronization asymmetry in the $\theta$ and $\alpha$ bands showed statistically significant differences between responders and non-responders in the OFF (ANOVA, $p=0.01$ ) and ON (ANOVA, $p=0.002)$ conditions. In the $\theta$ band, mean hemispheric synchronization asymmetry (Equation 8) showed a statistically significant difference between responders and non-responders in the $\mathrm{ON}$ condition ( $p=0.03$, corrected for multiple comparisons). Although the OFF condition showed a similar trend, it did not reach statistical significance (Figure 4a). Healthy controls showed a trend similar to responders but opposite to non-responders, although the result did not reach statistical significance (Figure $4 \mathrm{~b}$ ). Mean hemispheric synchronization in the $\alpha$ band did not reach statistical significance.

As mean hemispheric synchronization asymmetry revealed a difference between responders and non-responders in the $\theta$ band, we pursued this by displaying the correlation of frontal and parietal mean synchronization with the HAMD-17 score change across all 12 subjects. Figure 5 shows the results. A positive correlation was seen in frontal mean synchronization asymmetry in the OFF $(r=0.21)$ and $\mathrm{ON}(r=0.38)$ conditions, whereas a negative correlation was seen in parietal mean synchronization asymmetry in the OFF $(r=-0.68)$ and ON $(r=-0.70)$ conditions.

\section{Connectivity Matrices and Network Diagrams}

We further investigated the underlying network architecture. Figure 6a (top) shows the statistically significant $(p<0.05$ on a Kruskal-Wallis test) node-to-node synchronization values where responders showed higher connectivity than non-responders, whereas Figure 6a (bottom) shows the same where non-responders showed higher connectivity than responders. The corresponding network diagram is plotted in Figure 6b. Responders show stronger cross-hemispheric connectivity, particularly between right frontal/central and left parietal channels. Non-responders, on the other hand, show higher connectivity from the right parietal channels to frontal channels, as well as higher cross-hemispheric connectivity in the parietal and parietooccipital regions that is largely left-right symmetric. A higher overall number of edges can be observed in the ON than the OFF condition.

\section{DISCUSSION}

Although our findings should be considered preliminary given the small patient sample, we found robust and consistent, statistically significant differences in neural dynamics as revealed by EEG power and synchronization, between individuals with a history of MDD who showed a clinical response to DBS and those who did not. Furthermore, patterns seen in responders were similar to those seen in healthy controls. Below we elaborate on the details of these findings.

\section{Power Asymmetry}

Frontal $\theta$ oscillations are implicated in emotion processing, as well as other brain functions that are affected by depression including attention, learning, and memory (Bekkedal et al, 2011; Knyazev, 2007). Although most depression research has focused on the frontal and anterior 

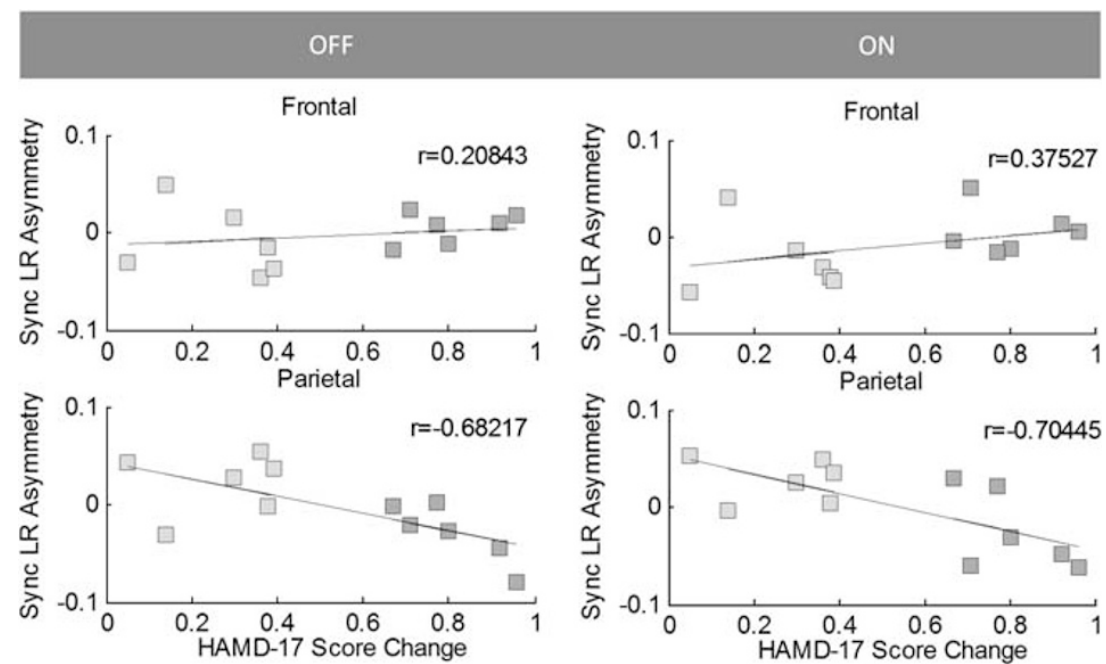

Figure 5 Top: correlation between frontal $\theta$ left-right synchronization asymmetry and HAMD- 17 scores for the OFF (left) and ON (right) conditions. Bottom: correlation between parietal $\theta$ left-right synchronization asymmetry and HAMD- 17 scores for the OFF (left) and ON (right) conditions.
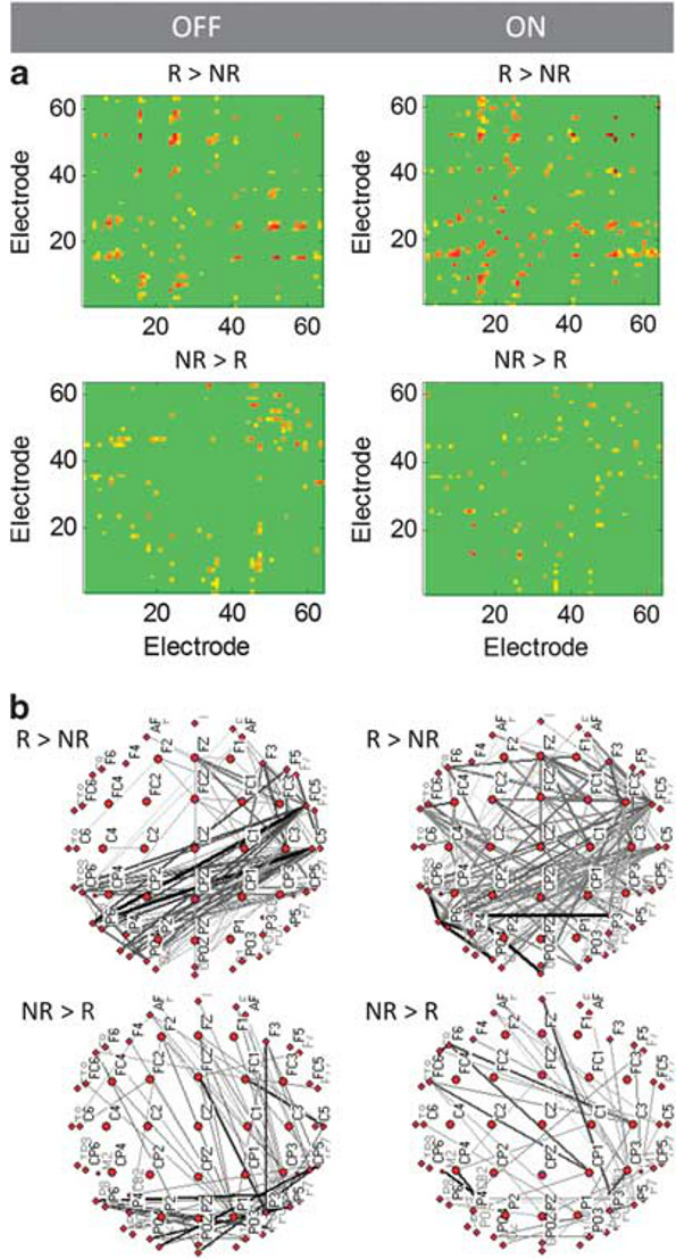

Figure 6 (a) Group-averaged connectivity matrices in the $\theta$ band for $R>N R$ (top row) and NR>R (bottom row) for the OFF (left) and ON (right) condition. The connectivity values are thresholded at $p<0.05$ using a Kruskal-Wallis test. (b) Network diagrams corresponding to the connectivity matrices in panel a. cingulate cortex (Mayberg et al, 1997; Salvadore et al, 2009; Salvadore et al, 2010), the parietal cortex also has been implicated in emotional deficits (Liotti and Mayberg, 2001; Mayberg, 1997). EEG parietal $\alpha$ asymmetry results have been mixed, but generally point to an increase in leftrelative-to-right $\alpha$ power in depressed patients (Thibodeau et al, 2006).

Consequently, we created a measure of asymmetry (Equation 2 in the Appendix 1) that combines frontal $\theta$ and parietal $\alpha$ asymmetry taking into account the left-right inverted nature of their relationship in these two frequency bands, which we termed hemispheric asymmetry, $A_{\mathrm{H}}$. This measure revealed statistically significant electrophysiological differences between responders and non-responders in the OFF and ON conditions. The OFF and ON conditions were very similar for each group indicating little changes in the underlying electrophysiological dynamics when the DBS electrodes were turned on. The lack of an acute stimulation effect is compatible with previous findings that mood symptoms improve across a 3-to-6-month interval following initial stimulation and show little impact of brief discontinuation after reaching a plateau (Lozano et al, 2008; Mayberg et al, 2005). These lasting neural changes have been attributed to neural plasticity (Lujan et al, 2008) as well as physiological changes (Kalbe et al, 2009) and molecular alterations that may involve adaptive gene expressions (Schulte et al, 2006).

An investigation of frontal $\theta$ asymmetry with the HAMD17 scores in patients shows that score improvement after DBS surgery correlates with an increase in left-relative-toright activity (Figure 3). In other words, an improvement in the HAMD-17 scores correlated with the reversal in the value of frontal asymmetry observed in MDD patients. Improvement in the HAMD-17 scores after surgery correlated with a reversal in the value of parietal $\alpha$ asymmetry observed in MDD patients. These trends were similar for the OFF and ON conditions. These findings are consistent with various studies that show an inverse 
relationship between parietal $\alpha$ oscillations and cortical activity in other frequency bands (typically $\theta$ and gamma $(\gamma)$ ), where an increase in parietal $\alpha$ activity in the left hemisphere relative to the right is associated with a decrease in cortical activity in the left hemisphere relative to the right in these frequency bands and vice versa. Some recent task experiments have shown that $\alpha$ oscillations have a role in the disengagement of brain regions that are not necessary for the task (Haegens et al, 2010; Mathewson et al, 2009; van Ede et al, 2011).

Although frontal $\alpha$ asymmetry is widely reported in the literature as a biomarker of depression, not all studies that looked for this asymmetry in depressed patients were able to reproduce it. Of the 26 studies compiled by Thibodeau et al (2006), only 10 studies showed a statistically significant $(p<0.05)$ frontal $\alpha$ asymmetry. It is therefore not surprising that we did not observe a statistically significant result in our small cohort of patients.

Overall, our results indicate that despite modest improvements in their HAMD-17 scores, non-responders show the same asymmetries that have been reported in currently depressed patients, namely, a combination of a decrease in left-relative-to-right frontal activity in the $\theta$ band and an increase in left-relative-to-right parietal activity in the $\alpha$ band. Importantly, our results suggest that the effect has been reversed in responders who show an asymmetry similar to healthy controls. The change in frontal and parietal asymmetry implicates global network effects in the context of local stimulation (ie, long-range functional connectivity changes), as the DBS electrodes are a long distance away from these regions. Electric fields decay exponentially with distance from the tip of the DBS electrode and, hence, only neurons in the immediate surroundings of the electrodes are stimulated directly.

\section{Synchronization Asymmetry}

Two primary observations require neural networks to explain the mechanism at play in DBS dynamics. First, the fact that a number of different DBS targets are able to effectively relieve depression symptoms; and second, changes in brain dynamics in frontal and parietal regions that are well beyond the short range over which the electric fields from the DBS electrodes have any direct effect on neural currents. Indeed, in this study, statistically significant differences in functional connectivity were found between responders and non-responders in the $\theta$ band in the ON condition with a similar trend in the OFF condition that did not reach statistical significance. In particular, nonresponders showed higher left-relative-to-right parietal synchronization than responders. Synchronization asymmetry correlated with after-surgery improvement in the HAMD-17 scores for parietal $\theta$ in both the ON and OFF conditions (Figure 5). These findings indicate an increase in left-relative-to-right connectivity in non-responders that gets reversed after DBS surgery in responders who show an asymmetry similar to controls (Figure 5).

Despite evidence pointing to functional connectivity as a potentially important biomarker of depression, only a handful of EEG functional connectivity studies currently exist in the literature. Furthermore, these studies have used different approaches and methodologies to investigate differences in brain dynamics between depressed patients and healthy controls making it difficult to compare outcomes. For example, Lee et al (2011) used correlations between power series of channel pairs as a measure of connectivity; Leistedt et al (2009) used synchronization likelihood (Stam et al, 2003); whereas Fingelkurts et al (2007) used an in-house synchronization measure termed index of structural synchronization (Fingelkurts and Kahkonen, 2005). Although these and other EEG/MEG measures of connectivity (phase coherency, phase lag index, imaginary coherency, etc) have been shown to capture aspects of correlations/synchronization between two time series, they are known to perform differently. Several factors contribute to this variation in performance including: whether they measure power correlation, phase synchronization or a mixture of both; whether they capture non-linear as well as linear dependencies; and whether they are able to detect full connectivity over the entire $2 \pi$ phase range. Furthermore, these measures are subject to different systematic effects that contribute to accumulated errors; not least among them is the well-known problem of volume conduction. Because of these and other confounding complex factors, one has to be particularly cautious when comparing across connectivity results from different studies.

\section{Network Diagrams}

We constructed network diagrams for synchronization in the $\theta$ band for the ON and OFF conditions. The most obvious pattern differentiating responders from nonresponders that emerged from these diagrams was seen in the OFF condition in long-range connections between right parietal and left frontal/central connections (Figure 6b, $\mathrm{R}>\mathrm{NR}$ ). Although these differences are still observed in the ON condition, other widespread connectivity differences between responders and non-responders are observed. This is not particularly surprising, as the DBS stimulation would necessarily lead to instantaneous effects that may not be associated with anti-depressive effects. This result points to right parietal to left frontal/central connectivity as a potential biomarker of response after DBS surgery, and potential response to anti-depressive effects (eg, pharmacological treatment of MDD) in general. This is a key finding that highlights the posterior regions as part of the depression network, which had typically focused on the limbic-frontal regions.

\section{Confounds and Limitations}

All patients were on multiple antidepressant medications at the time of surgery. Generally, the medications at the time of the recording changed with some medications discontinued, new medications introduced and dosage changed either upward or downward. Although antidepressant medications can impact the observed EEG signals, no obvious trend in medication changes can be seen that would differentiate responders from non-responders, suggesting that the impact of such a confound would be small.

Another potential confound results from differences in the time interval between the onset of DBS chronic stimulation and the EEG recording between responders and non-responders. This time interval was $47 \pm 22$ months 
for responders and $33 \pm 7$ months for non-responders. As the variations are quite large, the difference between the two groups is not significant and would likely result in a small confounding factor on our results.

In this experiment, the EEG recording for the OFF condition commenced minutes after the DBS stimulation was turned off. Often a delay of $30-60 \mathrm{~min}$ is introduced to allow for the chronic effects of stimulation to resolve. It remains unclear, however, whether a delay is needed, and if so how long this delay should be to allow for the transient effects of stimulation to decay. This remains a potential confound that we were not able to quantify.

Finally, the lack of baseline data limits our ability to assert whether the observed differences between responders and non-responders existed in baseline data making them biomarkers that predict response or whether such differences occurred as a result of chronic DBS stimulation, thereby suggesting a mechanism of action. Comparison of responders and non-responders to healthy controls sheds some light on this issue as EEG rhythms were similar between responders and healthy controls suggesting that chronic DBS stimulation might have resulted in normalization of EEG rhythms in the response group. Future studies where comparisons with baseline data are conducted can better answer this question.

Although some of these confounds (medication changes, time elapsed between DBS onset and EEG recording) can in principle be accounted for in the analysis, the small sample size precludes such determination. One must therefore be cautious in the interpretation of our findings. Future experiments with larger sample size would help confirm these results and aid in their interpretation.

\section{Conclusions}

In this study, we assessed differences in brain dynamics as recorded by EEG between responders and non-responders after DBS surgery with the DBS electrodes on and off. Despite the small patient sample size, we found robust and consistent, statistically significant findings in neural dynamics, as revealed in EEG power and synchronization, between individuals with a history of MDD who showed a clinical response to DBS and those who did not. Unfortunately, we could not compare current findings against individual patients' baseline data to directly evaluate changes related to chronic DBS stimulation, but we can draw conclusions in relation to the patterns observed in a cohort of healthy controls. These findings revealed important facts about the role of DBS in alleviating depressive symptoms: 1) DBS stimulation has a role in normalizing spectral rhythms in brain regions associated with depressive symptoms. These mechanisms include the $\theta$ rhythm in frontal regions that is likely related to brain engagement, but also disengagement mechanisms that manifest as $\alpha$ oscillations in the EEG spectral rhythms. 2) Long-range functional connectivity has a central role in depression, particularly between left frontal/ central regions and right parietal regions, which DBS stimulation serves to normalize as well. This is a key finding that highlights the posterior regions as part of the depression network, which had typically focused on the limbic-frontal regions. Further studies that build on these findings by further exploring the role of posterior regions in MDD, particularly in the context of disengagement mechanisms would be of tremendous value in elucidating the electrophysiological mechanisms associated with MDD. Future MEG studies can shed some light on the anatomical origin of these rhythms and reveal differences in connectivity between anatomical regions (as opposed to EEG scalp electrodes). Finally, analysis of effective connectivity may help reveal the directional flow of information between these regions, thereby pinpointing anatomical locations that may serve as more effective DBS targets.

\section{FUNDING AND DISCLOSURE}

This work was supported by the McDonnell Foundation, the Ontario Mental Health Foundation (OMHF), the Canadian Institutes of Health Research (CIHR), the Brain and Behaviour Research Foundation and the Temerty Family and Grant Family and through the Centre for Addiction and Mental Health (CAMH) Foundation and the Campbell Institute. ZJD received external funding through Brainsway, a travel allowance through Pfizer and Merck, speaker funding through Sepracor Inc., Astra Zeneca and served on the advisory board for Hoffmann-La Roche Limited. PG served on the advisory committee or a similar body for Eli Lilly Canada, conducted studies for Brain Cells, Clera, GSK, and St Jude Medical, received honoraria or other fees from Astra Zeneca, BMS, Pfizer, Eli Lilly, and St Jude Medical. AML is a consultant for Ceregene, Medtronic, Boston Scientific, and St Jude. He is a board member and receives compensation from Functional Neuroscience. SHK has acted as advisor/consultant or speaker for AA Pharma, Abbott Laboratories, Astra Zeneca, BristolMyers Squibb, Eli Lilly, Elsevier, Forest Laboratories, Glia Communications, Indigene Lifesystems, Lundbeck, Lundbeck Institute, Pfizer, Spimaco, Symbiote Medical Communications and Spirant Communications. He has received grant support from Bristol-Myers Squibb, Brain Cells, Clera, Eli Lilly, Lundbeck, Ontario Brain Institute, Pfizer, Servier and St Jude Medical. MAQ, ABP, CWT and MPM have nothing to disclose.

\section{REFERENCES}

Anderson RJ, Frye MA, Abulseoud OA, Lee KH, McGillivray JA, Berk $M$ et al (2012). Deep brain stimulation for treatmentresistant depression: efficacy, safety and mechanisms of action. Neurosci Biobehav Rev 36: 1920-1933.

Babiloni C, De Pandis MF, Vecchio F, Buffo P, Sorpresi F, Frisoni $\mathrm{GB}$ et al (2011). Cortical sources of resting state electroencephalographic rhythms in Parkinson's disease related dementia and Alzheimer's disease. Clin Neurophysiol 122: 2355-2364.

Bares M, Brunovsky M, Kopecek M, Novak T, Stopkova P, Kozeny $\mathrm{J}$ et al (2008). Early reduction in prefrontal theta QEEG cordance value predicts response to venlafaxine treatment in patients with resistant depressive disorder. Eur Psychiatry 23: 350-355.

Bekkedal MY, Rossi J 3rd, Panksepp J (2011). Human brain EEG indices of emotions: delineating responses to affective vocalizations by measuring frontal theta event-related synchronization. Neurosci Biobehav Rev 35: 1959-1970.

Bosboom JL, Stoffers D, Wolters E, Stam CJ, Berendse HW (2009). MEG resting state functional connectivity in Parkinson's disease related dementia. J Neural Transmission 116: 193-202.

Broadway JM, Holtzheimer PE, Hilimire MR, Parks NA, Devylder JE, Mayberg HS et al (2012). Frontal theta cordance predicts 6month antidepressant response to subcallosal cingulate deep 
brain stimulation for treatment-resistant depression: a pilot study. Neuropsychopharmacology 37: 1764-1772.

Bullmore E, Sporns O (2009). Complex brain networks: graph theoretical analysis of structural and functional systems. Nat Rev Neurosci 10: 186-198.

Cornwell BR, Johnson LL, Holroyd T, Carver FW, Grillon C (2008). Human hippocampal and parahippocampal theta during goaldirected spatial navigation predicts performance on a virtual Morris water maze. J Neurosci 28: 5983-5990.

Coryell W, Nopoulos P, Drevets W, Wilson T, Andreasen NC (2005). Subgenual prefrontal cortex volumes in major depressive disorder and schizophrenia: diagnostic specificity and prognostic implications. Am J Psychiatry 162: 1706-1712.

de Haan W, van der Flier WM, Wang H, Van Mieghem PF, Scheltens P, Stam CJ (2012). Disruption of functional brain networks in Alzheimer's disease: what can we learn from graph spectral analysis of resting-state magnetoencephalography? Brain Connect 2: 45-55.

Fingelkurts AA, Kahkonen S (2005). Functional connectivity in the brain-is it an elusive concept? Neurosci Biobehav Rev 28: 827-836.

Fingelkurts AA, Rytsala H, Suominen K, Isometsa E, Kahkonen S (2007). Impaired functional connectivity at EEG alpha and theta frequency bands in major depression. Hum Brain Mapp 28: 247-261.

Frysinger RC, Quigg M, Elias WJ (2006). Bipolar deep brain stimulation permits routine EKG, EEG, and polysomnography. Neurology 66: 268-270.

Giacobbe P, Mayberg HS, Lozano AM (2009). Treatment resistant depression as a failure of brain homeostatic mechanisms: implications for deep brain stimulation. Exp Neurol 219: 44-52.

Greenberg BD, Price LH, Rauch SL, Friehs G, Noren G, Malone D et al (2003). Neurosurgery for intractable obsessive-compulsive disorder and depression: critical issues. Neurosurg Clin N Am 14: 199-212.

Guevara R, Velazquez JL, Nenadovic V, Wennberg R, Senjanovic G, Dominguez LG (2005). Phase synchronization measurements using electroencephalographic recordings: what can we really say about neuronal synchrony? Neuroinformatics 3: 301-314.

Haegens S, Luther L, Jensen O (2012). Somatosensory anticipatory alpha activity increases to suppress distracting input. J Cognitiv Neurosci 24: 677-685.

Haegens S, Osipova D, Oostenveld R, Jensen O (2010). Somatosensory working memory performance in humans depends on both engagement and disengagement of regions in a distributed network. Hum Brain Mapp 31: 26-35.

Hamani C, Mayberg H, Snyder B, Giacobbe P, Kennedy S, Lozano AM (2009). Deep brain stimulation of the subcallosal cingulate gyrus for depression: anatomical location of active contacts in clinical responders and a suggested guideline for targeting. J Neurosurg 111: 1209-1215.

Hamani C, Mayberg H, Stone S, Laxton A, Haber S, Lozano AM (2011). The subcallosal cingulate gyrus in the context of major depression. Biol Psychiatry 69: 301-308.

Hanslmayr S, Backes H, Straub S, Popov T, Langguth B, Hajak G et al (2012). Enhanced resting-state oscillations in schizophrenia are associated with decreased synchronization during inattentional blindness. Hum Brain Mapp 34: 2266-2275.

Hinkley LB, Vinogradov S, Guggisberg AG, Fisher M, Findlay AM, Nagarajan SS (2011). Clinical symptoms and alpha band restingstate functional connectivity imaging in patients with schizophrenia: implications for novel approaches to treatment. Biol Psychiatry 70: 1134-1142.

Holtzheimer PE 3rd, Mayberg HS (2010). Deep brain stimulation for treatment-resistant depression. Am J Psychiatry 167: 1437-1444.

Iosifescu DV, Greenwald S, Devlin P, Perlis RH, Denninger JW, Alpert JE et al (2008). Pretreatment frontal EEG and changes in suicidal ideation during SSRI treatment in major depressive disorder. Acta psychiatrica Scandinavica 117: 271-276.

Jalili M, Lavoie S, Deppen P, Meuli R, Do KQ, Cuenod M et al (2007). Dysconnection topography in schizophrenia revealed with state-space analysis of EEG. PloS One 2: e1059.
Jimenez F, Velasco F, Salin-Pascual R, Hernandez JA, Velasco M, Criales JL et al (2005). A patient with a resistant major depression disorder treated with deep brain stimulation in the inferior thalamic peduncle. Neurosurgery 57: 585-593 discussion 585-593.

Kalbe E, Voges J, Weber T, Haarer M, Baudrexel S, Klein JC et al (2009). Frontal FDG-PET activity correlates with cognitive outcome after STN-DBS in Parkinson disease. Neurology 72: 42-49.

Kayser S, Bewernick BH, Grubert C, Hadrysiewicz BL, Axmacher N, Schlaepfer TE (2011). Antidepressant effects, of magnetic seizure therapy and electroconvulsive therapy, in treatmentresistant depression. J Psychiatr Res 45: 569-576.

Kennedy SH, Konarski JZ, Segal ZV, Lau MA, Bieling PJ, McIntyre $\mathrm{RS}$ et al (2007). Differences in brain glucose metabolism between responders to $\mathrm{CBT}$ and venlafaxine in a 16-week randomized controlled trial. Am J Psychiatry 164: 778-788.

Kessler RC, Berglund P, Demler O, Jin R, Merikangas KR, Walters EE (2005a). Lifetime prevalence and age-of-onset distributions of DSM-IV disorders in the National Comorbidity Survey Replication. Arch Gen Psychiatry 62: 593-602.

Kessler RC, Chiu WT, Demler O, Merikangas KR, Walters EE (2005b). Prevalence, severity, and comorbidity of 12-month DSM-IV disorders in the National Comorbidity Survey Replication. Arch Gen Psychiatry 62: 617-627.

Kessler RC, Merikangas KR, Wang PS (2007). Prevalence, comorbidity, and service utilization for mood disorders in the United States at the beginning of the twenty-first century. Annu Rev Clin Psychol 3: 137-158.

Knyazev GG (2007). Motivation, emotion, and their inhibitory control mirrored in brain oscillations. Neurosci Biobehav Rev 31: 377-395.

Kwon JS, Youn T, Jung HY (1996). Right hemisphere abnormalities in major depression: quantitative electroencephalographic findings before and after treatment. J Affect Disord 40: 169-173.

Lakhan SE, Callaway E (2010). Deep brain stimulation for obsessive-compulsive disorder and treatment-resistant depression: systematic review. BMC Res Notes 3: 60.

Laxton AW, Neimat JS, Davis KD, Womelsdorf T, Hutchison WD, Dostrovsky JO et al (2013). Neuronal coding of implicit emotion categories in the subcallosal cortex in patients with depression. Biol Psychiatry 74: 714-719.

Lee TW, Wu YT, Yu YW, Chen MC, Chen TJ (2011). The implication of functional connectivity strength in predicting treatment response of major depressive disorder: a resting EEG study. Psychiatry Res 194: 372-377.

Leistedt SJ, Coumans N, Dumont M, Lanquart JP, Stam CJ, Linkowski P (2009). Altered sleep brain functional connectivity in acutely depressed patients. Hum Brain Mapp 30: 2207-2219.

Linkenkaer-Hansen K, Monto S, Rytsala H, Suominen K, Isometsa E, Kahkonen S (2005). Breakdown of long-range temporal correlations in theta oscillations in patients with major depressive disorder. J Neurosci 25: 10131-10137.

Liotti M, Mayberg HS (2001). The role of functional neuroimaging in the neuropsychology of depression. J Clin Exp Neuropsychol 23: $121-136$

Lozano AM, Giacobbe P, Hamani C, Rizvi SJ, Kennedy SH, Kolivakis TT et al (2012). A multicenter pilot study of subcallosal cingulate area deep brain stimulation for treatment-resistant depression. J Neurosurg 116: 315-322.

Lozano AM, Mayberg HS, Giacobbe P, Hamani C, Craddock RC, Kennedy SH (2008). Subcallosal cingulate gyrus deep brain stimulation for treatment-resistant depression. Biol Psychiatry 64: 461-467.

Lujan JL, Chaturvedi A, McIntyre CC (2008). Tracking the mechanisms of deep brain stimulation for neuropsychiatric disorders. Front Biosci 13: 5892-5904.

Lyoo IK, Kim MJ, Stoll AL, Demopulos CM, Parow AM, Dager SR et al (2004). Frontal lobe gray matter density decreases in bipolar I disorder. Biol Psychiatry 55: 648-651. 
Malone DA Jr., Dougherty DD, Rezai AR, Carpenter LL, Friehs GM, Eskandar EN et al (2009). Deep brain stimulation of the ventral capsule/ventral striatum for treatment-resistant depression. Biol Psychiatry 65: 267-275.

Mathewson KE, Gratton G, Fabiani M, Beck DM, Ro T (2009). To see or not to see: prestimulus alpha phase predicts visual awareness. J Neurosci 29: 2725-2732.

Mayberg HS (1997). Limbic-cortical dysregulation: a proposed model of depression. J Neuropsychiatry Clin Neurosci 9: 471-481.

Mayberg HS (2003). Modulating dysfunctional limbic-cortical circuits in depression: towards development of brain-based algorithms for diagnosis and optimised treatment. Br Med Bull 65: 193-207.

Mayberg HS, Brannan SK, Mahurin RK, Jerabek PA, Brickman JS, Tekell JL et al (1997). Cingulate function in depression: a potential predictor of treatment response. Neuroreport 8: 1057-1061.

Mayberg HS, Liotti M, Brannan SK, McGinnis S, Mahurin RK, Jerabek PA et al (1999). Reciprocal limbic-cortical function and negative mood: converging PET findings in depression and normal sadness. Am J Psychiatry 156: 675-682.

Mayberg HS, Lozano AM, Voon V, McNeely HE, Seminowicz D, Hamani C et al (2005). Deep brain stimulation for treatmentresistant depression. Neuron 45: 651-660.

Mormann F, Andrzejak RG, Kreuz T, Rieke C, David P, Elger CE et al (2003). Automated detection of a preseizure state based on a decrease in synchronization in intracranial electroencephalogram recordings from epilepsy patients. Phys Rev E, Stat Nonlin Soft Matter Phys 67(2 Pt 1): 021912.

Mormann F, Lehnertz K, David P, Elger CE (2000). Mean phase coherence as a measure for phase synchronization and its application to the EEG of epilepsy patients. Phys D 144: 358-369.

Neimat JS, Hamani C, Giacobbe P, Merskey H, Kennedy SH, Mayberg HS et al (2008). Neural stimulation successfully treats depression in patients with prior ablative cingulotomy. Am J Psychiatry 165: 687-693.

Nitschke JB, Heller W, Palmieri PA, Miller GA (1999). Contrasting patterns of brain activity in anxious apprehension and anxious arousal. Psychophysiology 36: 628-637.

Nock MK, Hwang I, Sampson NA, Kessler RC (2010). Mental disorders, comorbidity and suicidal behavior: results from the National Comorbidity Survey Replication. Mol Psychiatry 15: 868-876.

Nugent AC, Milham MP, Bain EE, Mah L, Cannon DM, Marrett S et al (2006). Cortical abnormalities in bipolar disorder investigated with MRI and voxel-based morphometry. NeuroImage 30: 485-497.

Nunez PL, Srinivasan R, Westdorp AF, Wijesinghe RS, Tucker DM, Silberstein RB et al (1997). EEG coherency. I: Statistics, reference electrode, volume conduction, Laplacians, cortical imaging, and interpretation at multiple scales. Electroencephalograph Clin Neurophysiol 103: 499-515.

Rosenblum MG, Pikovsky AS, Kurths J (1996). Phase synchronization of chaotic oscillators. Phys Rev Lett 76: 1804.

Salvadore G, Cornwell BR, Colon-Rosario V, Coppola R, Grillon C, Zarate CA Jr. et al (2009). Increased anterior cingulate cortical activity in response to fearful faces: a neurophysiological biomarker that predicts rapid antidepressant response to ketamine. Biol Psychiatry 65: 289-295.

Salvadore G, Cornwell BR, Sambataro F, Latov D, Colon-Rosario V, Carver F et al (2010). Anterior cingulate desynchronization and functional connectivity with the amygdala during a working memory task predict rapid antidepressant response to ketamine. Neuropsychopharmacology 35: 1415-1422.

Sartorius A, Kiening KL, Kirsch P, von Gall CC, Haberkorn U, Unterberg AW et al (2010). Remission of major depression under deep brain stimulation of the lateral habenula in a therapy-refractory patient. Biol Psychiatry 67: e9-e11.

Schlaepfer TE, Bewernick BH, Kayser S, Madler B, Coenen VA (2013). Rapid effects of deep brain stimulation for treatmentresistant major depression. Biol Psychiatry 73: 1204-1212.
Schlaepfer TE, Cohen MX, Frick C, Kosel M, Brodesser D, Axmacher $\mathrm{N}$ et al (2008). Deep brain stimulation to reward circuitry alleviates anhedonia in refractory major depression. Neuropsychopharmacology 33: 368-377.

Schulte T, Brecht S, Herdegen T, Illert M, Mehdorn HM, Hamel W (2006). Induction of immediate early gene expression by highfrequency stimulation of the subthalamic nucleus in rats. Neuroscience 138: 1377-1385.

Seminowicz DA, Mayberg HS, McIntosh AR, Goldapple K, Kennedy S, Segal Z et al (2004). Limbic-frontal circuitry in major depression: a path modeling metanalysis. NeuroImage 22: 409-418.

Stam CJ, Breakspear M, van Cappellen van Walsum AM, van Dijk BW (2003). Nonlinear synchronization in EEG and whole-head MEG recordings of healthy subjects. Hum Brain Mapp 19: 63-78.

Stam CJ, de Haan W, Daffertshofer A, Jones BF, Manshanden I, van Cappellen van Walsum AM et al (2009). Graph theoretical analysis of magnetoencephalographic functional connectivity in Alzheimer's disease. Brain 132(Pt 1): 213-224.

Stam CJ, Nolte G, Daffertshofer A (2007a). Phase lag index: assessment of functional connectivity from multi channel EEG and MEG with diminished bias from common sources. Hum Brain Mapp 28: 1178-1193.

Stam CJ, Reijneveld JC (2007b). Graph theoretical analysis of complex networks in the brain. Nonlin Biomed Phys 1: 3.

Stewart JL, Towers DN, Coan JA, Allen JJ (2011). The oft-neglected role of parietal EEG asymmetry and risk for major depressive disorder. Psychophysiology 48: 82-95.

Stoffers D, Bosboom JL, Deijen JB, Wolters E, Stam CJ, Berendse HW (2008). Increased cortico-cortical functional connectivity in early-stage Parkinson's disease: an MEG study. NeuroImage 41: 212-222.

Tahaei M, Jalili M, Knyazeva M (2012). Synchronizability of EEGbased functional networks in early Alzheimer's disease. IEEE Trans Neural Syst Rehabil Eng 20: 636-641.

Tass P, Rosenblum MG, JWeule J, Kurths J, Pikovsky AS, Volkmann A et al (1998). Detection of n:m phase locking from noisy data: application to magnetoencephalography. Phys Rev Lett 81: 3291-3294.

Tesche CD, Karhu J (2000). Theta oscillations index human hippocampal activation during a working memory task. Proc Natl Acad Sci USA 97: 919-924.

Thibodeau R, Jorgensen RS, Kim S (2006). Depression, anxiety, and resting frontal EEG asymmetry: a meta-analytic review. J Abnorm Psychol 115: 715-729.

Urakami Y (2008). Relationships between sleep spindles and activities of cerebral cortex as determined by simultaneous EEG and MEG recording. J Clin Neurophysiol 25: 13-24.

van Dijk H, Schoffelen JM, Oostenveld R, Jensen O (2008). Prestimulus oscillatory activity in the alpha band predicts visual discrimination ability. J Neurosci 28: 1816-1823.

van Ede F, de Lange F, Jensen O, Maris E (2011). Orienting attention to an upcoming tactile event involves a spatially and temporally specific modulation of sensorimotor alpha- and betaband oscillations. J Neurosci 31: 2016-2024.

van Gerven M, Jensen O (2009). Attention modulations of posterior alpha as a control signal for two-dimensional braincomputer interfaces. J Neurosci Methods 179: 78-84.

Williams DR, Gonzalez HM, Neighbors H, Nesse R, Abelson JM, Sweetman J et al (2007). Prevalence and distribution of major depressive disorder in African Americans, Caribbean blacks, and non-Hispanic whites: results from the National Survey of American Life. Arch Gen Psychiatry 64: 305-315.

Yang LM, Hu B, Xia YH, Zhang BL, Zhao H (2008). Lateral habenula lesions improve the behavioral response in depressed rats via increasing the serotonin level in dorsal raphe nucleus. Behav Brain Res 188: 84-90. 


\section{Appendix 1}

\section{Power asymmetry}

Frontal power asymmetry, $A_{\mathrm{F}}$, in the $\theta$ band was defined using channels $\mathrm{F} 3$ and $\mathrm{F} 4$ where

$$
A_{\mathrm{F}}=\frac{P(\mathrm{~F} 3)-P(\mathrm{~F} 4)}{P(\mathrm{~F} 3)+P(\mathrm{~F} 4)}
$$

A parietal $\alpha$ power asymmetry, $A_{\mathrm{P}}$, was defined similarly using channels P3 and P4. Note that odd number locations are sensors on the left hemisphere and even numbered are on the right hemisphere.

Hemispheric asymmetry computed as the weighted sum of frontal $\theta$ left-right power difference and parietal $\alpha$ rightleft power difference was defined as

$$
A_{\mathrm{H}}=\frac{P(\mathrm{~F} 3)-P(\mathrm{~F} 4)}{W_{\mathrm{F}}}+\frac{P(\mathrm{P} 4)-P(\mathrm{P} 3)}{W_{\mathrm{P}}}
$$

where

$$
W_{\mathrm{F}}=P(\mathrm{~F} 3)+P(\mathrm{~F} 4) \text {, }
$$

and

$$
W_{\mathrm{P}}=P(\mathrm{P} 3)+P(\mathrm{P} 4)
$$

\section{Functional connectivity}

For a given signal $s(t)$, with a Hilbert transform $s^{\prime}(t)$, the instantaneous phase difference $\phi(t)$ between two time series (labeled as a and b) is given by

$$
\begin{aligned}
\Delta \phi(t) & =\phi_{\mathrm{a}}(t)-\phi_{\mathrm{b}}(t) \\
& =\tan ^{-1}\left(\frac{s_{\mathrm{a}}^{\prime}(t) s_{\mathrm{b}}(t)-s_{\mathrm{a}}(t) s_{\mathrm{b}}^{\prime}(t)}{s_{\mathrm{a}}(t) s_{\mathrm{b}}(t)+s_{\mathrm{a}}^{\prime}(t) s_{\mathrm{b}}^{\prime}(t)}\right)
\end{aligned}
$$

and is confined to the interval $[0,2 \pi]$. Following Mormann et al (2000), we use a mean phase coherency measure of synchronization defined as

$$
R=\left|\frac{1}{N} \sum_{j}^{N-1} e^{i \Delta \phi(j \Delta t)}\right|
$$

where $j$ is the sample number and $N$ is the total number of samples. Mean phase coherency takes on values between 0 and 1, indicating no synchronization and full synchronization, respectively.

In this study, we computed phase coherence, $S_{i j}$, between all pairs of electrodes, then computed a mean synchronization, $S_{j}^{\text {mean }}$, for each electrode as

$$
S_{j}^{\text {mean }}=\frac{1}{N} \sum_{i=1, i \neq j}^{N} S_{i j}
$$

where $N=64$ is the total number of electrodes. Using this mean synchronization, we computed a mean synchronization asymmetry in the frontal, parietal/central regions shown in Figure $4 \mathrm{~d}$. For example, the mean frontal synchronization asymmetry, $S_{\mathrm{F}}^{\mathrm{A}}$, is given by

$S_{\mathrm{F}}^{\mathrm{A}}=\frac{\sum_{e=\mathrm{FL}} S_{e}-\sum_{e=\mathrm{FR}} S_{e}}{\sum_{e=\mathrm{FL}} S_{e}+\sum_{e=\mathrm{FR}} S_{e}}$

where $\mathrm{FL}=\{\mathrm{F} 1, \mathrm{~F} 3, \mathrm{~F} 5, \mathrm{FC} 1, \mathrm{FC} 3, \mathrm{FC} 5\} \quad$ and $\mathrm{FR}=\{\mathrm{F} 2, \mathrm{~F} 4$, $\mathrm{F} 6, \mathrm{FC} 2, \mathrm{FC} 4, \mathrm{FC} 6\}$. A similar formula was used for the parietal asymmetry with $\mathrm{PL}=\{\mathrm{CP} 1, \mathrm{CP} 3, \mathrm{CP} 5, \mathrm{P} 1, \mathrm{P} 3, \mathrm{P} 5\}$ and $\quad \mathrm{PR}=\{\mathrm{CP} 2, \mathrm{CP} 4, \mathrm{CP} 6, \mathrm{P} 2, \mathrm{P} 4, \mathrm{P} 6\} . \quad \mathrm{A}$ hemispheric synchronization asymmetry was defined to combine frontal and parietal asymmetry as was done for power asymmetry. 